\title{
Refractory Seizure with Autosomal Dominant Hypocalcemia with Hypercalciuria with a CASR Mutation Detected Using Whole Exome Sequencing
}

\author{
Seong Bae Ahn, MD', Sung Han Shim, MD², Eun-Gyong Yoo, MD¹, Mo Kyung Jung, MD', \\ Kyu Young Chae, MD ${ }^{1}$ \\ 'Department of Pediatrics, CHA Bundang Medical Center, CHA University, Seongnam, Korea \\ ${ }^{2}$ Genetic Laboratory, Fertility Center of CHA Gangnam Medical Center, CHA University, Seoul, Korea
}

Received: September 7, 2020

Revised: October 12, 2020

Accepted: October 12, 2020

Corresponding author:

Kyu Young Chae, MD

Department of Pediatrics, CHA

Bundang Medical Center, CHA

University, 59 Yatap-ro, Bundang-

gu, Seongnam 13496, Korea

Tel: +82-31-780-5230

Fax: +82-31-780-5239

E-mail: danielchae21@gmail.com
Autosomal-dominant hypocalcemia with hypercalciuria $(\mathrm{ADHH})$ is a genetic disease characterized by hypoparathyroidism, hypocalcemia, and varying degrees of hypocalcemia. Although convulsions can occur with hypocalcemia, most patients are asymptomatic or show intermittent limb numbness, muscular contractions, and laryngeal spasms [1]. Most patients with $\mathrm{ADHH}$ have a missense mutation in the calcium sensing receptor (CASR) gene [1]. CaSR is a 1078-residue glycoprotein, encoded by six exons of CASR located on chromosome 3q13.3-21, and is primarily expressed in the parathyroid gland and renal tubules [2]. It plays a pivotal role in systemic calcium metabolism by regulating parathyroid hormone secretion and urinary calcium excretion [3]. Dysfunctions in the CaSR manifest differentially, depending on the locus of the mutation, or mutations in other CASR-associated genes [3].

Here, we report a case of $\mathrm{ADHH}$ as confirmed by whole exome sequencing (WES). A 9-yearold boy reported with frequent repetitive generalized tonic-clonic seizures over a course of 2 months. The seizures occurred spontaneously, lasting for 5 to 60 seconds, followed by urination and decreased muscle tone. He was born via cesarean section at 36 weeks gestation with a birth weight of $3.4 \mathrm{~kg}$ (94th percentile), and was subsequently admitted to the neonatal intensive care unit with hypoxia. Following discharge, he survived normally without any abnormal neurological symptoms. His mother had mild tingling sensations in her limbs, but was never diagnosed with any neurological conditions. At the first hospital visit, his body weight, height, and head circumference were $43.2 \mathrm{~kg}$ (86th percentile), $143.7 \mathrm{~cm}$ (82th percentile), and $56 \mathrm{~cm}$ (96th percentile), respectively. He had no physical defects or deformities. His initial vital signs were within the normal ranges, and neurologic examination was unremarkable. Upon laboratory testing, hypocalcemia $(6.6 \mathrm{mg} / \mathrm{dL}$; reference range, 8.6 to 10.2$)$ and hyperphosphatemia $(8.8 \mathrm{mg} /$ $\mathrm{dL}$; reference range, 2.0 to 5.5 ) with borderline hypercalciuria (urine calcium/creatinine ratio: 0.198; reference range, 0 to 0.2 ) were found without evidence of severe vitamin $\mathrm{D}$ deficiency (25-hydroxy-vitamin D: $25.57 \mathrm{ng} / \mathrm{mL}$; reference range, 30 to 100). The serum levels of parathyroid hormone (PTH-I) dropped to $13.91 \mathrm{pg} /$ $\mathrm{mL}$ (reference range, 15 to 65 ). Interictal electro-

Copyright (C) 2021 Korean Child Neurology Society

This is an Open Access article distributed under the terms of the Creative Commons Attribution Non-Commercial License (http://creativecommons.org/licenses/by-nc/4.0/) which permits unrestricted non-commercial use, distribution, and reproduction in any medium, provided the original work is properly cited. 
encephalography (EEG) showed semi-rhythmic high amplitude 3 to $4 \mathrm{~Hz}$ delta frequency activity from the left fronto-temporal area following hyperventilation. Under the initial impression of hypoparathyroidism, oral calcium preparations $(40 \mathrm{mg} / \mathrm{kg} /$ day as elemental calcium) and alfacalcidol ( $12 \mathrm{ng} / \mathrm{kg} /$ day) were prescribed. His serum calcium levels recovered, and were within the normal range. A year later, the patient revisited the hospital because the frequency of convulsions that had previously decreased with medication, had increased. His serum calcium levels dropped to $6.8 \mathrm{mg} /$
dL despite high-dose calcium treatment. Intravenous calcium gluconate and high-dose vitamin D were injected, but refractory hypocalcemia with generalized seizures persisted. Brain computed tomography (CT) revealed multiple gross calcifications in the bilateral cerebral white matter, basal ganglia, thalamus, and cerebellum (Fig. 1A and B). A kidney stone was found on abdominopelvic CT scan (Fig. 1C). WES was performed for elucidating the exact cause of the symptoms, which revealed a likely pathogenic [4] heterozygous missense mutation in the CASR gene (c.721G $>$ A $[\mathrm{p}$.
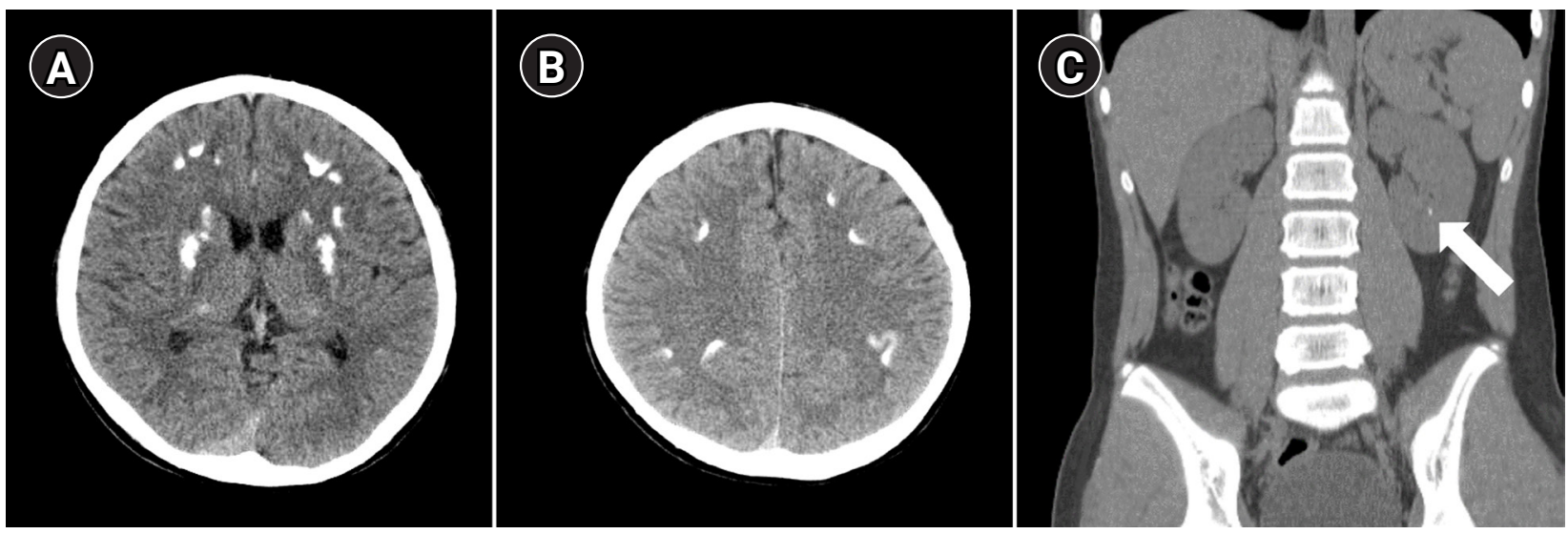

Fig. 1. (A, B) Multiple calcifications (white areas) are observed in the basal ganglia and frontal white matter in the cross sectional view on brain computed tomography. (C) A small kidney stone is evident in the left kidney (arrow).

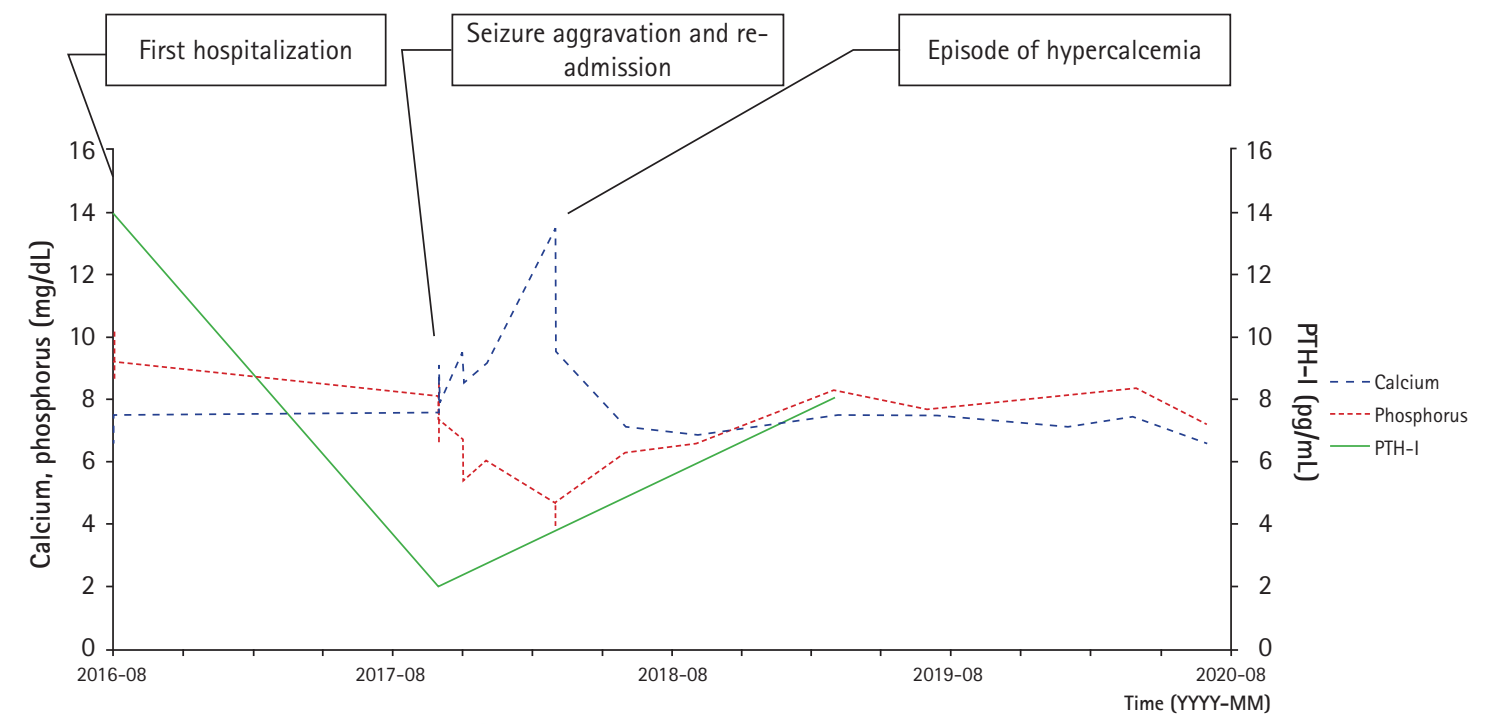

Seizure

PO calcium

$\mathrm{mg} / \mathrm{kg} /$ day)

Alfacalcido

(ng/kg/day)

Calcitriol

$(\mathrm{ng} / \mathrm{kg} / \mathrm{day})$

Thiazide

(mg/kg/day)

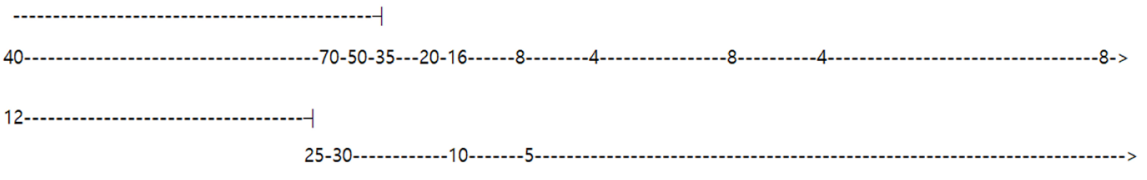

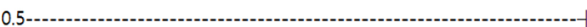

Fig. 2. The serial progress in the serum levels of calcium, phosphorus, and parathyroid hormone (PTH-I), and the doses of the medications. PO, peros. 
Glu241Lys]). Trio-WES was performed on the parents, and the same mutation was found in his mother. Blood tests were subsequently performed on his mother, and her serum levels of calcium and PTH-I were $6.6 \mathrm{mg} / \mathrm{dL}$ and $12.38 \mathrm{pg} / \mathrm{mL}$, respectively, indicating hypocalcemic hypoparathyroidism like the patient. The frequency of hypocalcemic seizures gradually decreased following treatment with increasing doses of calcitriol and calcium carbonate and the addition of $0.5 \mathrm{mg} / \mathrm{kg} /$ day hydrochlorothiazide. After 5 months, the patient was re-admitted with abrupt hypercalcemia. The levels of blood calcium and ionized calcium were $13.5 \mathrm{mg} / \mathrm{dL}$ and $1.68 \mathrm{mmol} / \mathrm{L}$, respectively, despite steadily decreasing doses of oral calcium supplementation. After the doses of calcium and vitamin $\mathrm{D}$ were further reduced, the blood calcium levels gradually returned to the normal range. An EEG after 16 months revealed no epileptic discharges. Three years post diagnosis, the patient was still taking calcitriol ( $5 \mathrm{ng} / \mathrm{kg} /$ day) and calcium carbonate ( $8 \mathrm{mg} /$ $\mathrm{kg}$ /day as elemental calcium), and did not have tingling sensations or seizures. The serial progression in the serum levels of calcium, phosphorus, and PTH-I, and dose of the medications are schematically presented in Fig. 2.

The common clinical symptoms of patients with hypoparathyroidism range from asymptomatic to severe symptomatic hypocalcemia. However, in this case, the clinical manifestations of a missense mutation in the CASR gene (c.721G > A [p.Glu241Lys]) had more diverse effects on the serum calcium levels, ranging from refractory hypocalcemia to hypercalcemia during long-term follow-up. Hyperparathyroidism or hypoparathyroidism may appear depending on the locus of the mutation. However, Hannan et al. [4] reported that both hypocalcemia and hypercalcemia can occur due to a p.Glu250Lys mutation in CASR. Similarly, the calcium levels in our patient remained normal for a while, for which the administration of calcium was gradually reduced. However, the patient suddenly developed severe hypercalcemia. Notably, the p. Glu241Lys mutation identified herein also caused severe hypercalcemia when the calcium replacement was tapered. During the 3 years of follow-up, the p.Glu241Lys mutation in CASR responded poorly to calcium or vitamin $\mathrm{D}$ modulation therapy and showed periodic dysregulated pendular hypocalcemia and hypercalcemia.

$\mathrm{ADHH}$ patients with hypocalcemic convulsions are commonly treated with parenteral calcium for increasing serum calcium, followed by maintenance with oral calcium and vitamin $\mathrm{D}$ supplementation. However, calcium treatments should be limited to hypocalcemic or symptomatic conditions, as nephrocalcinosis may occur as a side effect. Thiazides can be used for reducing the amount of excreted urinary calcium. The serum and urine calcium concentrations should be checked every 3 to 6 months for re-determining the appropriate dose of the drug [3]. In this case, severe hypocalcemia followed by hypercalcemia occurred within 5 months, despite regular checkups for calcium concentration and treatment modulation.

In conclusion, we report a case of a p.Glu241Lys mutation in CASR, as detected by WES, in which the serum calcium levels varied periodically between severe hypocalcemia with refractory generalized seizures and unexpected hypercalcemia during long-term follow-up. This is the first report of a p.Glu241Lys mutation in $C A S R$, with phenotypes of hypocalcemia and hypercalcemia, and insignificant responses to medical treatment. This mutation induces sudden irregular changes in the calcium concentration, making treatment difficult and requiring frequent serum calcium tests and detailed drug concentrations for controlling treatment.

This study was approved by the Institutional Review Board of CHA Bundang Medical Center (IRB No: 2018-06-008). Written informed consent was obtained from all patients.

\section{Conflicts of interest}

No potential conflict of interest relevant to this article was reported.

\section{ORCID}

Seong Bae Ahn, https:/ / orcid.org/0000-0002-1657-3247

Kyu Young Chae, https://orcid.org/0000-0003-3243-5853

\section{Author contribution}

Conceptualization: EGY and KYC. Methodology: SHS. Writing-original draft: SBA. Writing-review \& editing: EGY, MKJ, and KYC.

\section{References}

1. Moon JE, Lee SJ, Park SH, Kim J, Jin DK, Ko CW. De novo a novel variant of CaSR gene in a neonate with congenital hypoparathyroidism. Ann Pediatr Endocrinol Metab 2018;23:10711.

2. D'Souza-Li L, Yang B, Canaff L, Bai M, Hanley DA, Bastepe M, et al. Identification and functional characterization of novel calcium-sensing receptor mutations in familial hypocalciuric hypercalcemia and autosomal dominant hypocalcemia. J Clin Endocrinol Metab 2002;87:1309-18.

3. Vahe C, Benomar K, Espiard S, Coppin L, Jannin A, Odou MF, et al. Diseases associated with calcium-sensing receptor. Orphanet J Rare Dis 2017;12:19. 
4. Hannan FM, Nesbit MA, Zhang C, Cranston T, Curley AJ, Harding B, et al. Identification of 70 calcium-sensing receptor mutations in hyper- and hypo-calcaemic patients: evidence for clustering of extracellular domain mutations at calcium-binding sites. Hum Mol Genet 2012;21:2768-78. 